\title{
On the representation of regional characteristics by hydrographic measurements at central stations in four deep basins of the Baltic
} Sea

\author{
J. H. Reissmann \\ Institut für Ostseeforschung Warnemünde, Rostock, Germany \\ Received: 6 June 2005 - Published in Ocean Sci. Discuss.: 26 July 2005 \\ Revised: 19 April 2006 - Accepted: 21 June 2006 - Published: 20 July 2006
}

\begin{abstract}
In this work the eddy resolving data sets of salinity, temperature, and oxygen content aquired in the framework of the German-Russian project MESODYN (MESOscale DYNamics) in the Arkona Basin, the Bornholm Basin, the Stolpe Furrow, and the Eastern Gotland Basin during summer and winter stratification situations are utilized to examine to which extent the observations at the central monitoring stations within these basins are representative for the spatial mean state of the corresponding region with respect to comparative monitoring purposes of the whole Baltic Sea. The investigation covers profiles of salinity, potential temperature, oxygen content, potential density, and squared buoyancy or Brunt-Väisälä frequency. Moreover, some parameters of the halocline, namely its depth, thickness, and upper and lower boundaries, and the first baroclinic Rossby radii are subject to the investigation. The profiles match best for the squared buoyancy or Brunt-Väisälä frequency. The profiles of salinity match best in the Eastern Gotland Basin and worst in the Arkona Basin both for summer and winter stratification situations. The overall agreement for the halocline parameters is good. The baroclinic Rossby radii match their spatial mean values well, if the depth range considered for their calculation is restricted to the mean depth in each region at the bottom side. In doing so they also match the spatial mean values of the first baroclinic Rossby radii calculated considering the whole depth range at each station. Overall, the regional characteristics of the investigated quantities and parameters are represented well by the hydrographic measurements at the central stations in the four regions in spite of some significant differences between the spatial mean states and the observations at the central stations. In particular, the observations at the central stations seem to be usefull for
\end{abstract}

Correspondence to: J. H. Reißmann

(jan.reissmann@io-warnemuende.de) comparisons between these regions. However, the observed differences may affect regional investigations covering just a single region.

\section{Introduction}

The Baltic Sea is a semienclosed marginal sea, connected to the world ocean by the North Sea (e.g. Rodhe, 1998). The connection to the North Sea through the Kattegat and the Danish Straits is shallow and narrow resulting in a strongly suppressed water exchange between the Baltic and the North Sea. Moreover, the Baltic Sea itself is distinctly divided in basins and channels seperated by shallow sills. While precipitation and evaporation over the Baltic Sea are of the same order of magnitude on the annual scale, there is a large surplus of fresh water due to the huge drainage area of the Baltic Sea resulting in an outflow of fresh water at the surface. Contributions to the water balance of the Baltic Sea were estimated by HELCOM (1986), Bergström and Carlsson (1994), Lindau (2002), Hennemuth et al. (2003), and Omstedt and Nohr (2004), for example. A review is given by Omstedt et al. (2004). The loss of salt due to this outflow is compensated by incidental inflows of saline water from the North Sea. The inflowing water of high salinity and, consequently, high density spreads at the bottom into the western Baltic Sea. Depending on the magnitude of the inflow, the mixing, and regional stratification it adjusts accordingly to its density and follows a sequence of basins and channels into the Baltic proper.

As a consequence of the freshwater surplus in the Baltic Sea there are both a salinity gradient from the brackish waters in the western Baltic Sea to the nearly fresh waters in its northern parts and a strong permanent halocline, particularly in its deeper parts. The permanent halocline vigorously

Published by Copernicus GmbH on behalf of the European Geosciences Union. 


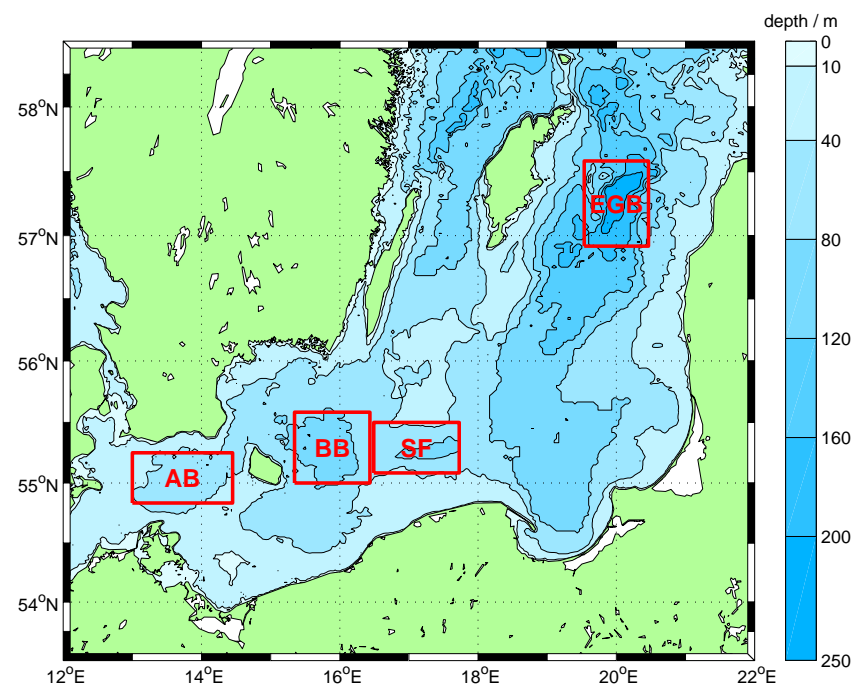

Fig. 1. Regions under investigation: AB: Arkona Basin, BB: Bornholm Basin, SF: Stolpe Furrow, EGB: Eastern Gotland Basin.

suppresses the vertical exchange of surface and bottom waters. Not even the convection in winter ranges deeper than the halocline. Therefore the bottom water below the halocline in the deep basins is transformed and, in particular, ventilated mainly by horizontal advection due to the inflows. This is the main reason for monitoring programmes such as the Baltic Monitoring Programme (BMP) or the Baltic Year (BY) to follow the inflow path along the sequence of basins and channels. Within these programmes the hydrographic conditions in the different basins are predominantly characterized by profiling measurements at one or a few single stations close to the center of each basin at their deepest locations.

Single hydrographic profiles from central locations were also utilized to describe regional characteristics in the Baltic Sea for other purposes than the mentioned monitoring. For example, Matthäus (1986) investigated the regional characteristics of the deep water in three basins of the Baltic Sea during stagnation periods using the time series of profiles resulting from one monitoring station in each considered basin. Kõuts and Omstedt (1993) analysed the deep water exchange and mixing properties in the Baltic proper by means of temperature and salinity profiles measured during the period from 1970 to 1990 at seven stations each representing one basin or pool in the employed approach of modelling the corresponding in- and outflows. Omstedt and Axell (1998) used temperature and salinity profiles from five stations located at central positions in five of 13 sub-basins for the validation of 15-year simulations modelling the Baltic Sea as 13 horizontally averaged sub-basins.

Although there is evidence of fluctuations inside the basins (e.g. Hagen and Feistel, 2004), all of these exemplified works have the basic assumption in common that single hydro- graphic profils measured at central stations in certain regions are representative for the regional conditions there. The purpose of this work is to examine to which extent this assumption is valid in four basins of the Baltic Sea utilizing threedimensional data fields of salinity, temperature, and oxygen content. Ideally, each of these data fields should represent the regional characteristics describing the overall condition in the respective area of investigation at a given time. Therefore their spatial coverage should be as high as possible resolving at least all relevant structures causing regional variations such as eddies or inflowing currents of different water masses with horizontal extensions in the order of a few baroclinic Rossby radii. On the other hand the time span for each complete survey should be as short as possible to get a nearly real snapshot of the hydrographic fields at the given time. At least the time span between each two stations should be shorter than the time a baroclinic wave needs to propagate along the distance between the corresponding two stations to minimize aliasing. Obviously, these requirements are contradicting and, consequently, it is impossible to aquire a data field perfectly meeting the needs for an ideal description of the regional characteristics. Balancing the need of a high coverage of the region with as much uniformly distributed stations as possible with the need of a time span for the survey as short as possible the utilized three-dimensional data fields were aquired quasi-synoptically with respect to the meteorological forcing using eddy resolving station grids. The data sets from the four basins, namely the Arkona Basin $(\mathrm{AB})$, the Bornholm Basin (BB), the Stolpe Furrow (SF), and the Eastern Gotland Basin (EGB) are considered seperately for summer and winter stratification situations.

\section{Data basis}

The German-Russian project MESODYN (MESOscale DYNamics) was initiated to investigate the spreading and transformation of dense deep water in the Baltic Sea. Therefore hydrographic data fields of salinity, temperature, and oxygen content were aquired using a standard CTD probe during 12 field campaigns in four deep basins of the Baltic Sea. The four basins were the $\mathrm{AB}, \mathrm{BB}, \mathrm{SF}$, and EGB. The exact regions under investigation are indicated in Fig. 1. Additionally, their accurate boundaries are given in Table 1 .

For all CTD surveys in each region the same regular station grid was applied. The respective four station grids are presented in Fig. 2. The horizontal spacing of each CTD survey was $2.5 \mathrm{~nm}(\approx 4.6 \mathrm{~km})$ in both zonal and meridional direction. The corresponding resolutions in degrees of latitude and longitude are given in Table 1. Fennel et al. (1991) calculated baroclinic Rossby radii in the order of magnitude of about $5 \mathrm{~km}$ for various regions of the Baltic Sea during different seasons. However, these regions were considerably larger than those investigated here and the hardly justified assumtion of a flat bottom was implied by taking the mean 
Table 1. Exact coordinates of the regions under investigation, the meridional resolution is $2.5^{\prime}$ for all four areas.

\begin{tabular}{cccccc}
\hline & \multicolumn{2}{c}{ Latitude } & \multicolumn{2}{c}{ Longitude } \\
Region & from & to & from & to & Resolution \\
\hline $\mathrm{AB}$ & $54^{\circ} \mathrm{N} 50^{\prime}$ & $55^{\circ} \mathrm{N} 15^{\prime}$ & $13^{\circ} \mathrm{E} 0^{\prime}$ & $14^{\circ} \mathrm{E} 27^{\prime}$ & $4.35^{\prime}$ \\
$\mathrm{BB}$ & $55^{\circ} \mathrm{N} 0^{\prime}$ & $55^{\circ} \mathrm{N} 35^{\prime}$ & $15^{\circ} \mathrm{E} 20.7^{\prime}$ & $16^{\circ} \mathrm{E} 26.2^{\prime}$ & $4.37^{\prime}$ \\
$\mathrm{SF}$ & $55^{\circ} \mathrm{N} 5^{\prime}$ & $55^{\circ} \mathrm{N} 30^{\prime}$ & $16^{\circ} \mathrm{E} 29.43^{\prime}$ & $17^{\circ} \mathrm{E} 43.67^{\prime}$ & $4.37^{\prime}$ \\
$\mathrm{EGB}$ & $56^{\circ} \mathrm{N} 55^{\prime}$ & $57^{\circ} \mathrm{N} 35^{\prime}$ & $19^{\circ} \mathrm{E} 32.1^{\prime}$ & $20^{\circ} \mathrm{E} 27.9^{\prime}$ & $4.65^{\prime}$ \\
\hline
\end{tabular}

\section{Arkona Basin}

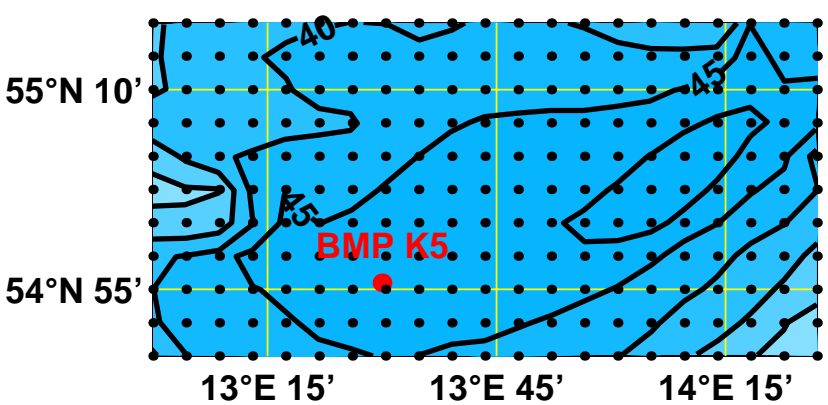

Bornholm Basin

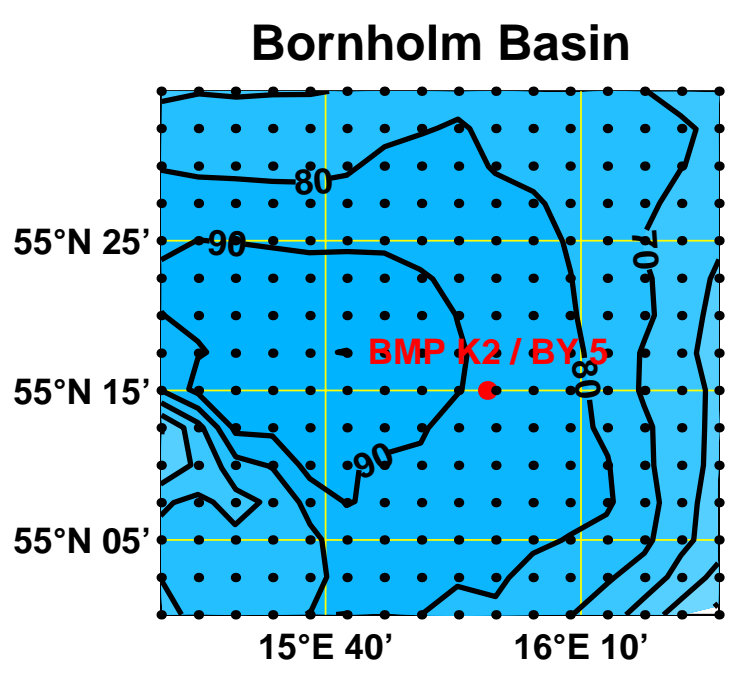

\section{Stolpe Furrow}

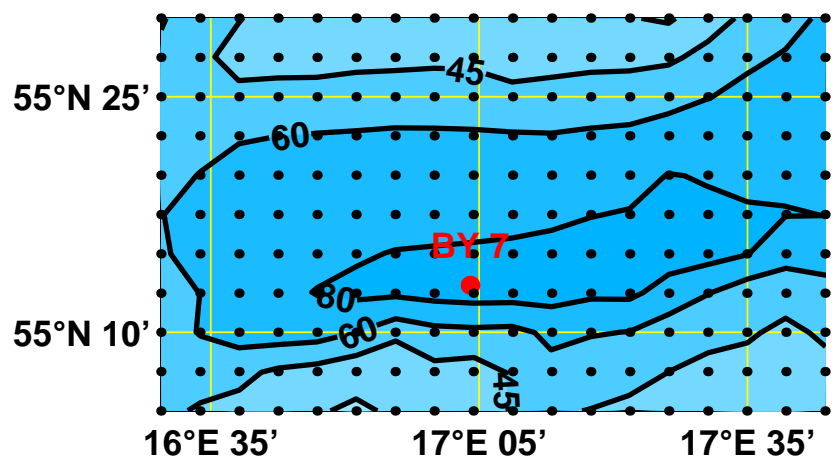

\section{Eastern Gotland Basin}

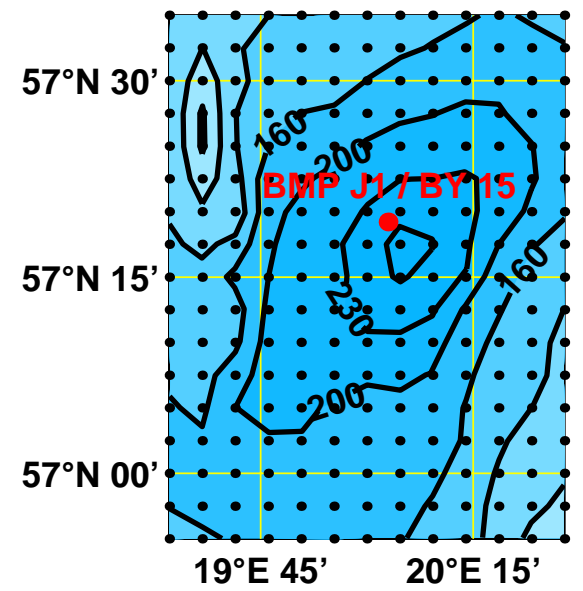

Fig. 2. Station grids of the four regions under investigation overlaid on contours of depth given in meters. Additionally, the BMP and BY monitoring stations considered as representative for the regions are indicated.

depth in each region as depth limit for the calculation of the baroclinic Rossby radii. Therefore larger baroclinic Rossby radii can be expected for the deeper central parts of these regions covering the areas considered here when the general increase of the baroclinic Rossby radius with increasing depth is taken into account. Accordingly, the most baroclinic eddies observed in the Baltic Sea are reported to have diameters between $10 \mathrm{~km}$ and $20 \mathrm{~km}$. With respect to these values the resulting data fields can be assumed as eddy resolving. Vertically the CTD profiles were sampled to 1 dbar corresponding to approximately $1 \mathrm{~m}$. This vertical resolution provides a sufficient reproduction of the vertical gradients in the halocline and thermocline.

The data acquisition times range between $92 \mathrm{~h}$ and $173 \mathrm{~h}$ for all surveys. On the occurrence of dramatic changes in the meteorological forcing the surveys were terminated. 
Table 2. Data sets from the AB.

\begin{tabular}{ccccc}
\hline Data set & Period & Duration & Stations & Stratification \\
\hline MD-04 & $04 / 12 / 1996-09 / 12 / 1996$ & $117 \mathrm{~h}$ & 231 & winter \\
MD-12 & $16 / 10 / 1999-20 / 10 / 1999$ & $92 \mathrm{~h}$ & 170 & winter \\
\hline
\end{tabular}

Table 3. Data sets from the BB.

\begin{tabular}{ccccc}
\hline Data set & Period & Duration & Stations & Stratification \\
\hline MD-03 & $08 / 09 / 1996-13 / 09 / 1996$ & $134 \mathrm{~h}$ & 176 & summer \\
MD-05 & $27 / 02 / 1997-06 / 03 / 1997$ & $173 \mathrm{~h}$ & 240 & winter \\
\hline
\end{tabular}

Table 4. Data sets from the SF.

\begin{tabular}{ccccc}
\hline Data set & Period & Duration & Stations & Stratification \\
\hline MD-01 & $02 / 03 / 1996-07 / 03 / 1996$ & $116 \mathrm{~h}$ & 192 & winter \\
MD-06 & $10 / 06 / 1997-14 / 06 / 1997$ & $96 \mathrm{~h}$ & 198 & summer \\
MD-09 & $07 / 11 / 1998-11 / 11 / 1998$ & $94 \mathrm{~h}$ & 176 & winter \\
\hline
\end{tabular}

Table 5. Data sets from the EGB.

\begin{tabular}{ccccc}
\hline Data set & Period & Duration & Stations & Stratification \\
\hline MD-02 & $11 / 06 / 1996-17 / 06 / 1996$ & $135 \mathrm{~h}$ & 208 & summer \\
MD-07 & $29 / 08 / 1997-04 / 09 / 1997$ & $151 \mathrm{~h}$ & 208 & summer \\
MD-08 & $19 / 04 / 1998-24 / 04 / 1998$ & $141 \mathrm{~h}$ & 195 & winter \\
MD-10 & $22 / 11 / 1998-27 / 11 / 1998$ & $104 \mathrm{~h}$ & 182 & winter \\
MD-11 & $19 / 08 / 1999-25 / 08 / 1999$ & $133 \mathrm{~h}$ & 221 & summer \\
\hline
\end{tabular}

Therefore each survey and resulting data fields can be considered as quasi-synoptic with respect to the synoptic meteorological forcing over the Baltic Sea with a time scale of three to six days. Admittedly, it was not possible to execute all stations of the respective station grid on every campaign because of the weather conditions and the cruise time schedules.

Each data set is assigned to a summer or winter situation according to the stratification with or without the thermocline in summer, respectively. An overview over the data aquisition times, the number of executed stations, and the stratification situations of the 12 data sets resulting from the MESODYN field campaigns is given in the Tables 2 to 5 by region.

In conclusion, the MESODYN data fields represent the regional characteristics in the respective areas of investigation at given times with respect to the requirements for this purpose given in Sect. 1 .

\section{Methods and results}

In Fig. 2 the positions of the BMP and BY monitoring stations which are considered as representative for the four regions are indicated. For each data set the station with the smallest ratio $d_{s t}^{\mathrm{mon}} / \Delta p_{s t}$ is determined. These stations are chosen as the central station of each data set having regard of both the distance $d_{s t}^{\text {mon }}$ from each station to the corresponding monitoring station and the depth range $\Delta p_{s t}$ covered by the profiles at each station. In most cases the central station is the closest station to the corresponding monitoring station (smallest $d_{s t}^{\text {mon }}$ ) in each data set as well due to the location of the monitoring stations in the vicinity of the deepest parts of the basins. The only exception occurs in data set MD-02. The central station of data set MD-02 is the second closest station to the monitoring station BMP J1/BY 15 which is the next station to the west of the closest one. The closest one is not chosen because the corresponding profiles only reach about half the way to the bottom due to technical problems at this station. The resulting distances $d_{c}^{\text {mon }}$ from the chosen central station to the corresponding monitoring station are listed in Table 7 for all data sets. 
Table 6. Vertical $r m s$ and $\bar{\sigma}$ parameters derived from the profiles of salinity $S$, potential temperature $T_{\text {pot }}$, oxygen content $o$, and potential density $\varrho_{\text {pot }}$ and the numbers of samples $n_{r m s}$ and $n_{\bar{\sigma}}$ used for the calculations of the $r m s$ and $\bar{\sigma}$, respectively, for all data sets.

\begin{tabular}{|c|c|c|c|c|c|c|c|c|c|c|c|c|}
\hline \multirow[b]{2}{*}{ Region } & \multirow[b]{2}{*}{ Season } & \multirow[b]{2}{*}{ Data set } & \multicolumn{2}{|c|}{$S / p s u$} & \multicolumn{2}{|c|}{$T_{\text {pot }} /{ }^{\circ} \mathrm{C}$} & \multicolumn{2}{|c|}{$o /(\mathrm{ml} / \mathrm{l})$} & \multicolumn{2}{|c|}{$\varrho_{\text {pot }} /\left(\mathrm{kg} / \mathrm{m}^{3}\right)$} & \multirow[b]{2}{*}{$n_{r m s}$} & \multirow[b]{2}{*}{$n_{\bar{\sigma}}$} \\
\hline & & & $r m s$ & $\bar{\sigma}$ & $r m s$ & $\bar{\sigma}$ & $\mathrm{rms}$ & $\bar{\sigma}$ & rms & $\bar{\sigma}$ & & \\
\hline \multirow[t]{2}{*}{$\mathrm{AB}$} & winter & MD-04 & 3.346 & 0.901 & 0.440 & 0.394 & 0.1 & 0.2 & 1.883 & 0.682 & 45 & 46 \\
\hline & winter & MD-12 & 2.769 & 1.369 & 0.203 & 0.581 & 0.2 & 0.6 & 1.566 & 1.031 & 45 & 47 \\
\hline \multirow[t]{2}{*}{$\mathrm{BB}$} & summer & MD-03 & 0.177 & 0.330 & 0.519 & 0.832 & 0.2 & 0.4 & 0.113 & 0.299 & 85 & 93 \\
\hline & winter & MD-05 & 1.221 & 0.576 & 0.672 & 0.710 & 0.7 & 0.7 & 0.708 & 0.435 & 86 & 93 \\
\hline \multirow[t]{3}{*}{ SF } & summer & MD-06 & 0.150 & 0.352 & 0.213 & 0.485 & 0.1 & 0.3 & 0.092 & 0.283 & 88 & 88 \\
\hline & winter & MD-01 & 0.899 & 0.577 & 0.840 & 0.572 & 1.1 & 0.7 & 0.527 & 0.442 & 84 & 88 \\
\hline & winter & MD-09 & 0.084 & 0.425 & 0.523 & 0.676 & 0.4 & 0.7 & 0.068 & 0.342 & 87 & 88 \\
\hline \multirow[t]{5}{*}{ EGB } & summer & MD-02 & 0.026 & 0.141 & 0.267 & 0.332 & 0.2 & 0.4 & 0.017 & 0.111 & 228 & 237 \\
\hline & summer & MD-07 & 0.001 & 0.097 & 0.136 & 0.239 & 0.0 & 0.3 & 0.003 & 0.085 & 234 & 237 \\
\hline & summer & MD-11 & 0.006 & 0.083 & 0.171 & 0.263 & 0.0 & 0.2 & 0.009 & 0.083 & 232 & 238 \\
\hline & winter & MD-08 & 0.021 & 0.103 & 0.021 & 0.164 & 0.0 & 0.3 & 0.012 & 0.076 & 234 & 237 \\
\hline & winter & MD-10 & 0.046 & 0.158 & 0.421 & 0.244 & 0.3 & 0.2 & 0.034 & 0.121 & 215 & 237 \\
\hline
\end{tabular}

Table 7. Distance $d_{c}^{\text {mon }}$ from the central station to the corresponding monitoring station, vertical $r m s$ and $\bar{\sigma}$ parameters derived from the profiles of squared buoyancy or Brunt-Väisälä frequency $N^{2}$, and the numbers of samples $n_{r m s}$ and $n_{\bar{\sigma}}$ used for the calculations of the $r m s$ and $\bar{\sigma}$, respectively, for all data sets.

\begin{tabular}{lllccccc}
\hline \multirow{2}{*}{ Region } & & \multicolumn{7}{c}{$N^{2} /\left(1 / 10^{6} \mathrm{~s}^{2}\right)$} \\
\hline \multirow{2}{*}{ AB } & Season & Data set & $d_{c}^{\mathrm{mon}} / \mathrm{km}$ & \multicolumn{1}{c}{$r m s$} & $\bar{\sigma}$ & $n_{r m s}$ & $n_{\bar{\sigma}}$ \\
& winter & MD-04 & 1.0 & 11.657 & 2015.851 & 43 & 44 \\
& winter & MD-12 & 1.0 & 5.317 & 2256.154 & 43 & 45 \\
& summer & MD-03 & 0.8 & 0.352 & 600.294 & 83 & 91 \\
& winter & MD-05 & 1.1 & 2.734 & 890.936 & 84 & 91 \\
SF & summer & MD-06 & 0.9 & 0.524 & 722.717 & 86 & 86 \\
& winter & MD-01 & 1.4 & 3.902 & 982.217 & 82 & 86 \\
& winter & MD-09 & 1.0 & 0.383 & 750.266 & 85 & 86 \\
EGB & summer & MD-02 & 3.3 & 0.019 & 111.344 & 226 & 235 \\
& summer & MD-07 & 1.9 & 0.036 & 147.111 & 232 & 235 \\
& summer & MD-11 & 2.3 & 0.188 & 176.361 & 230 & 236 \\
& winter & MD-08 & 1.9 & 0.026 & 97.594 & 232 & 235 \\
& winter & MD-10 & 2.3 & 0.041 & 123.434 & 179 & 234 \\
\hline
\end{tabular}

In this section the profiles and deduced quantities from the central stations are compared with the respective averages over all stations in the corresponding data set to examine to which extent the monitoring stations are representative for the considered regions. The differences are evaluated in terms of the respective standard deviations $\sigma$. Subject to comparison are some profiles of measured and derived hydrographic and physical quantities, parameters of the permanent halocline, and the first baroclinic Rossby radii.

\subsection{Profiles of hydrographic and physical quantities}

In a first step the profiles of salinity, potential temperature, and oxygen content are directly compared for one summer and one winter stratification situation for each region. The only exception is the $\mathrm{AB}$ for which no data set representing a summer stratification situation is available, see Table 2. As an example for a winter stratification situation in the $A B$ the data set MD-04 is chosen. For the BB exactly one data set is available for each of both situations, see Table 3 . For the SF exactly one data set is available for a summer stratification situation and data set MD-01 is taken as an example for a winter stratification situation, see Table 4 . For the EGB the data sets MD-11 and MD-08 are chosen to represent a summer and a winter stratification situation, respectively, see Table 5. The profiles of potential temperature with reference level $p_{\text {ref }}=0$ were calculated by solving the entropy conservation equation according to Feistel (2003) numerically by means of Newton iteration as proposed by Feistel (2005). The resulting plots are shown in the Figs. 3 to 6 by region. 

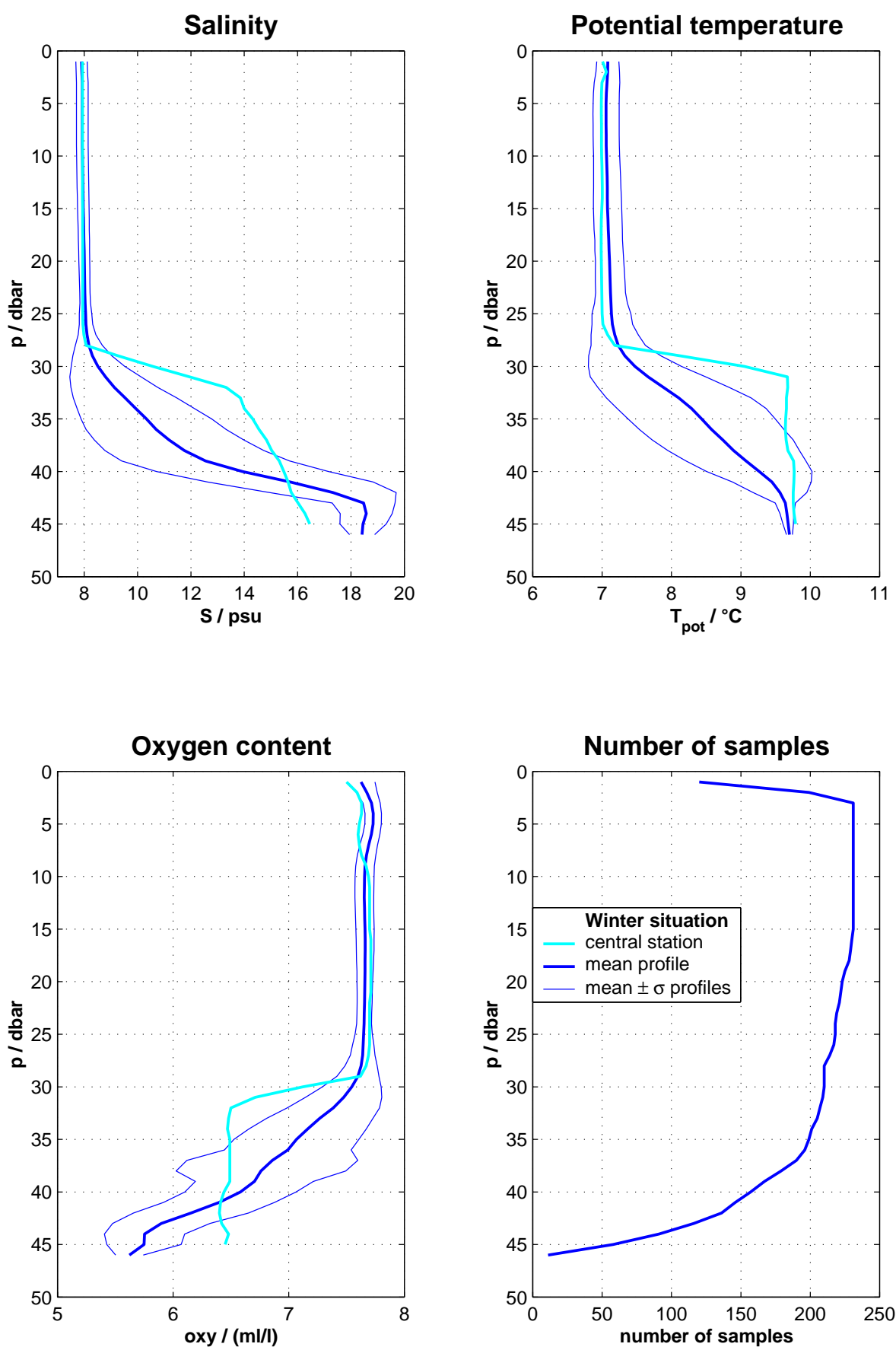

Fig. 3. Profiles of salinity, potential temperature, and oxygen content at the central station in comparison with the respective mean profiles accompanied by variation limits of one standard deviation $\sigma$ and the number of samples with respect to depth for a winter stratification situation in the $\mathrm{AB}$ (MD-04). No data set with a summer stratification situation in the $\mathrm{AB}$ is available.

These figures also include the information about the depth dependence of the number of samples. The legends given in the corresponding subplots are valid for all plots in the figures.

The average profiles over all stations in the corresponding data set, which are assumed to be representative for the hy- drographic conditions in the respective region, are chosen as the respective isobaric mean profiles here. Accordingly, the respective isobaric standard deviations are given to evaluate the differences between the profiles at the central stations and the corresponding average profiles. Obviously, the isobaric averaging is not the only choice. It is chosen as the most 

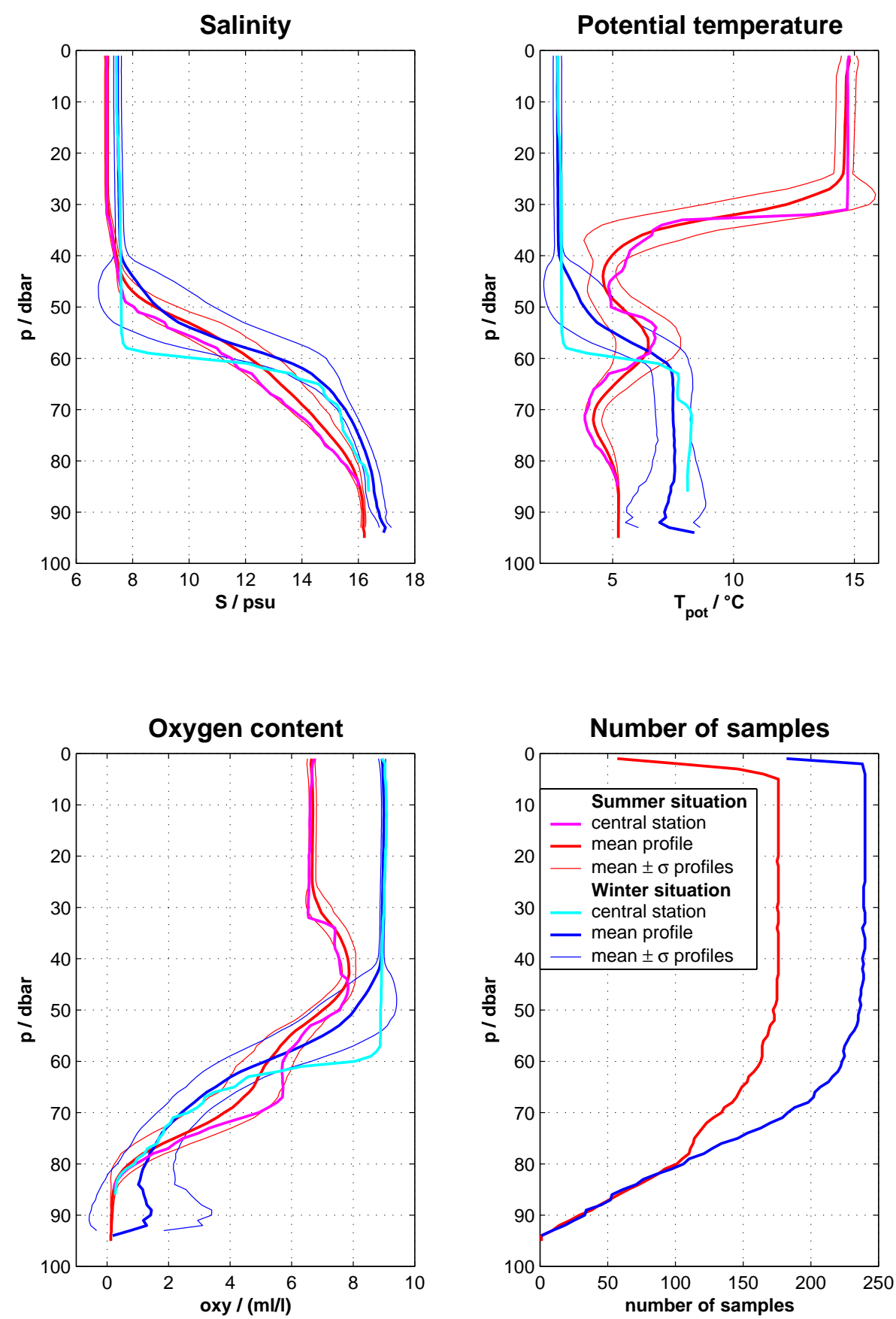

Fig. 4. Profiles of salinity, potential temperature, and oxygen content at the central station in comparison with the respective mean profiles accompanied by variation limits of one standard deviation $\sigma$ and the number of samples with respect to depth for a summer and a winter stratification situation in the BB (MD-03 and MD-05, respectively).

intuitive one because it keeps the depth co-ordinate given by the profiling CTD measurements. Moreover, the pressure directly corresponds to a spatial depth within the uncertainties of the profiling. On the other hand, the isobaric averageing causes a smoothing of the profiles. However, the definition of the representative profiles is reasonable if the isobaric variations are taken into account. For example, the smoothing of the mean profiles due to varying depths of the halocline in the region, which might be much sharper in each single profile than in the isobaric mean profile, is indicated by large variations in the depth range of the halocline compared to the rest of the profile. In the opposed case of a smooth isobaric mean profile with small variances in the range of the halocline a smooth halocline with nearly the same depth in the 

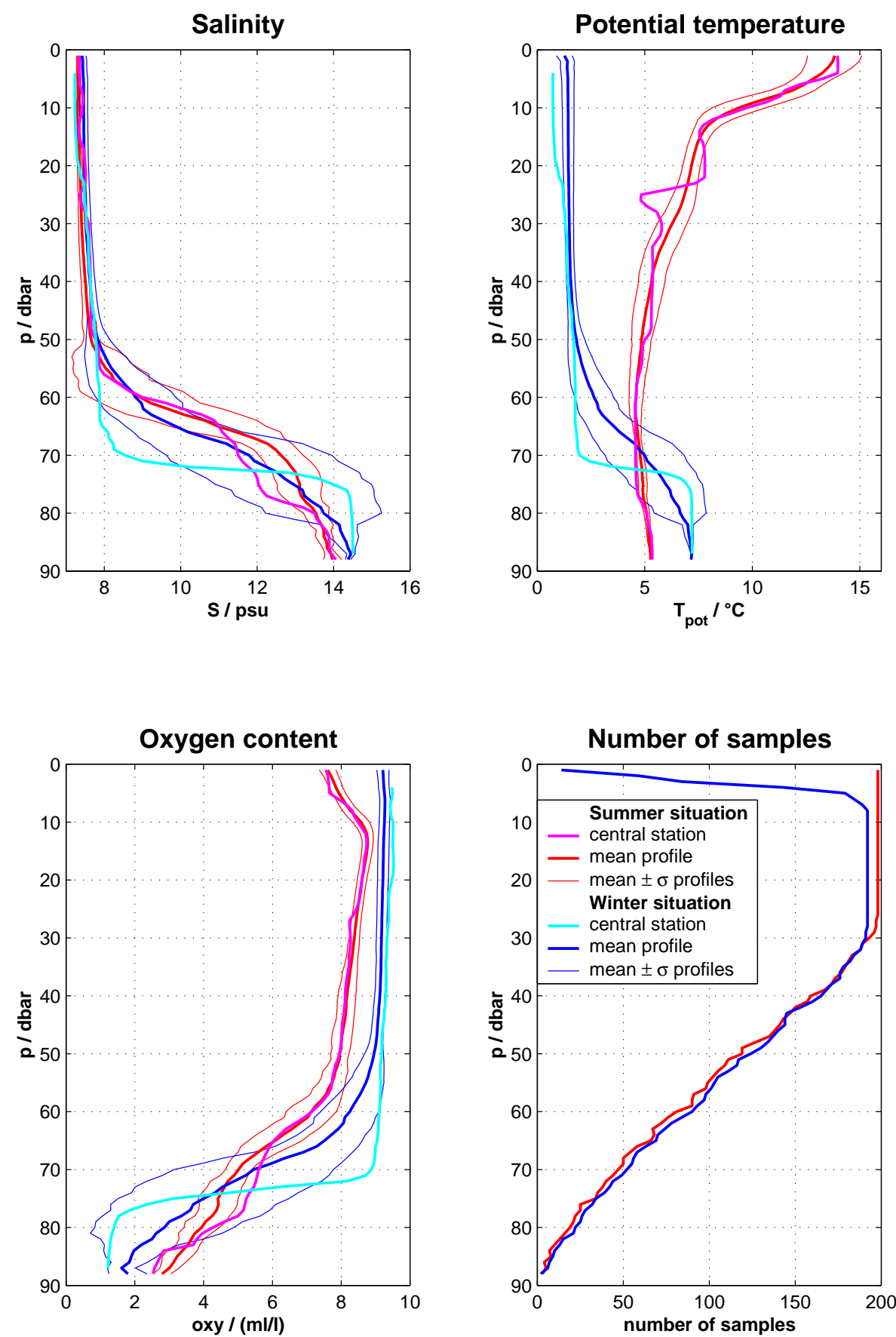

Fig. 5. Profiles of salinity, potential temperature, and oxygen content at the central station in comparison with the respective mean profiles accompanied by variation limits of one standard deviation $\sigma$ and the number of samples with respect to depth for a summer and a winter stratification situation in the SF (MD-06 and MD-01, respectively). Due to technical problems the profile of the oxygen content is missing at one station. Therefore the number of samples has to be reduced by one in the depth range from 4 dbar to 62 dbar for the oxygen content.

whole region would be indicated by isobaric standard deviations in the same order of magnitude over the whole depth range.

The Figs. 3 to 6 give a valuable visual impression of the differences between the profiles at the central station and the respective mean profiles and the isobaric variations within the regions, particularly of their vertical differences. But it is also reasonable to reduce these informations to a few numbers for reasons of clarity and to provide an easy approach for a quantitative comparison of the different data 

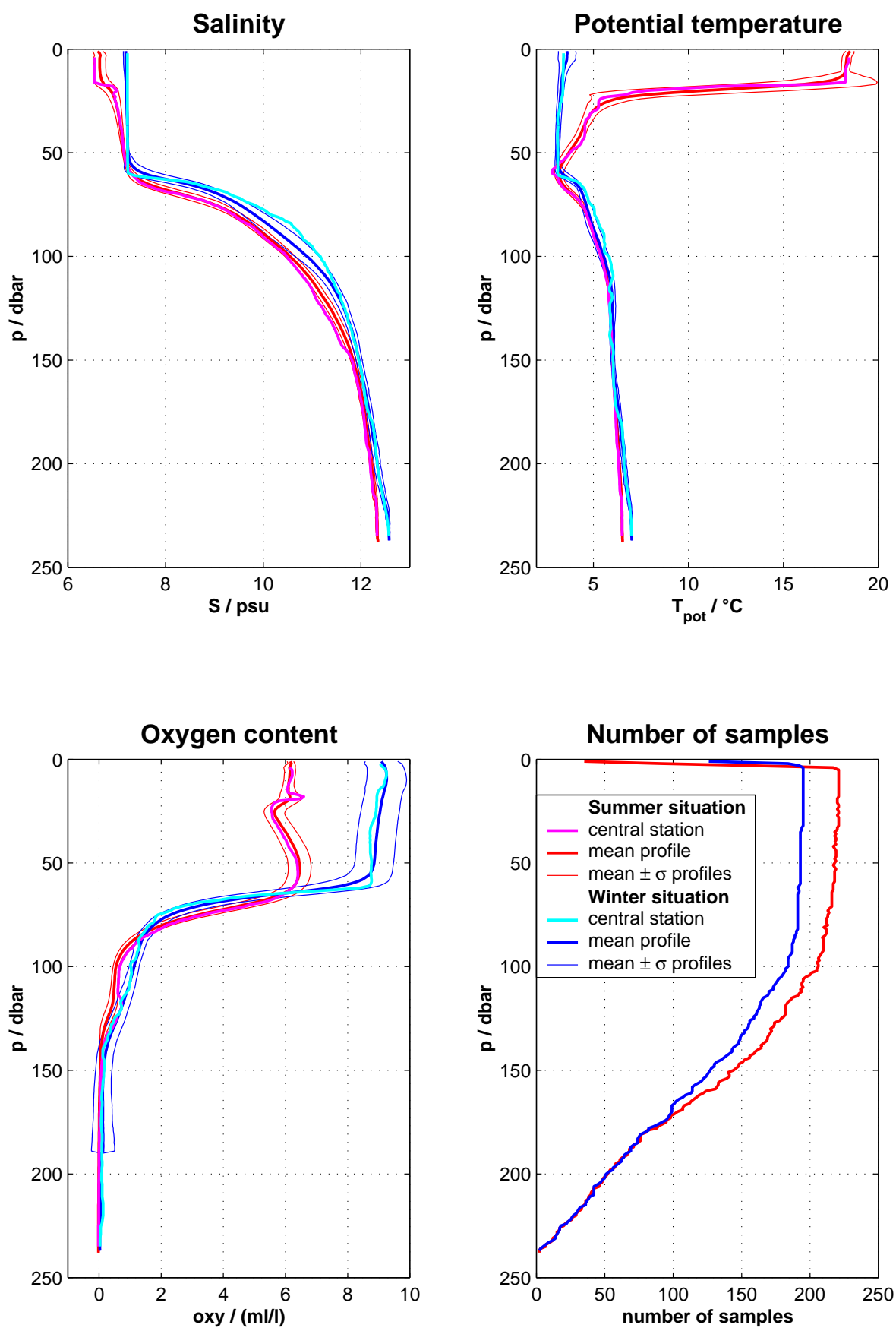

Fig. 6. Profiles of salinity, potential temperature, and oxygen content at the central station in comparison with the respective mean profiles accompanied by variation limits of one standard deviation $\sigma$ and the number of samples with respect to depth for a summer and a winter stratification situation in the EGB (MD-11 and MD-08, respectively).

sets. Therefore the vertical root mean squares $r m s$ of the deviations of the profiles at the central stations from the corresponding mean profiles were calculated as an intuitive estimate of a standardised isobaric overall deviation between these profiles. For comparison the vertical mean values $\bar{\sigma}$ of the isobaric standard deviations were calculated as an intuitive estimate of a standardised isobaric overall variation within the corresponding region. In Table 6 the results for the profiles of salinity $S$, potential temperature $T_{\text {pot }}$, oxygen content $o$, and potential density $\varrho_{\text {pot }}$ are given for all data sets. The profiles of $\varrho_{\text {pot }}$ with reference level $p_{\text {ref }}=0$ were calculated analogous to that of $T_{\text {pot }}$ before. In Table 7 the analogous results for $r m s$ and $\bar{\sigma}$ of the profiles of squared buoyancy or Brunt-Väisälä frequency $N^{2}$ are given for all 
data sets accompanied by the distances $d_{c}^{\text {mon }}$ from the respective central stations to the corresponding monitoring station.

Using the acceleration due to gravity $g$ the profiles of $N^{2}$ were calculated according to :

$N^{2}=-\frac{g}{\varrho_{\text {pot }}} \frac{d \varrho_{\text {pot }}}{d z}$

The derivation of $\varrho_{\text {pot }}$ with respect to the vertical, upward directed coordinate $z$ of the potential density profiles in Eq. (1) at each data point is determined by means of a linear least squares fit to the considered and its two directly neighbouring data points. If one or both of the neighbouring data points of one data point are missing in the profile no derivation is determined for that data point. Therefore the profiles of $N^{2}$ consist of at least two data points less than the other profiles because of the upper and the lower end of the profiles. This is also the reason why separate numbers of samples $n_{r m s}$ and $n_{\bar{\sigma}}$ used for the calculations of the $r m s$ and $\bar{\sigma}$, respectively, are given in Table 7 for the calculations concerning $N^{2}$. For the data set MD-10 the numbers $n_{r m s}$ and $n_{\bar{\sigma}}$ for $N^{2}$ differ by more than two from the respective numbers for the other quantities because the profiles aquired during the corresponding field campaign contain a couple of data gaps due to the hard weather conditions at that time. For example, each of the measured profiles at the central station contain 20 data gaps which are evenly distributed over the hole depth range fortunately. The corresponding profile of $N^{2}$ at the central station has even more data gaps due to the determination of the derivation of $\varrho_{\text {pot }}$. The difference between $n_{r m s}$ and $n_{\bar{\sigma}}$ for each data set originates from the different pressure levels covered by the profiles at the respective central station and the corresponding profiles of standard deviations.

\subsection{Parameters of the permanent halocline}

Four parameters describing the permanent halocline are derived from the salinity profiles. The four parameters are the depth $d$ of the permanent halocline, its upper and lower boundaries $u$ and $l$, respectively, and its thickness $\Delta d=l-u$. The depth $d$ is determined by means of the first derivation $d S / d z$ of the salinity $S$ with respect to the vertical, upward directed coordinate $z$ of the salinity profiles. It is defined as $d=-z$ by the location $z$ of the absolute minimum of $d S / d z$ at the local minima of $d S / d z$. In most cases this location coincides with the location of the absolute minimum of $d S / d z$. In other cases the absolute minimum of $d S / d z$ is located at the bottom and no local minima of $d S / d z$ exist. These cases are interpreted as situations without any permanent halocline and, according to its definition, no halocline depth $d$ is obtained for the respective profiles. If a halocline depth $d$ was found for a certain salinity profile, it is tried to determine the upper and lower boundary $u$ and $l$ of the halocline by means of the second derivation $d^{2} S / d z^{2}$ of the salinity $S$ with respect to the vertical coordinate $z$. Analogous to the halocline depth they are defined as $u=-z$ and $l=-z$ by the locations $z$ of the local maximum and minimum of $d^{2} S / d z^{2}$ which are the closest to $d$ in upward and downward direction, respectively. The thickness $\Delta d$ of the halocline is simply determined to $\Delta d=l-u$, if both boundaries $u$ and $l$ of the halocline could have been determined.

The derivations $d S / d z$ and $d^{2} S / d z^{2}$ were determined from the profiles of $S$ and $d S / d z$, respectively, in the same way as the derivation of the potential density $\varrho_{\text {pot }}$ with respect to the vertical coordinate $z$ in the context of Eq. (1) in Sect. 3.1. But the salinity profiles $S$ were linear interpolated before their first derivation $d S / d z$ was determined to avoid large data gaps within the profiles of $d S / d z$ and $d^{2} S / d z^{2}$. Moreover, the closest ten neighbouring data points to each data point (five to the top and five to the bottom) were used for the linear fits determining the derivations instead of only the closest two ones to get the derivations somewhat more smooth and less sensitve for small fluctuations in the salinity. This choice is appropriate for the determination of the halocline parameters because in this way the linear fits cover a depth range of the same order of magnitude as the thickness of the permanent halocline leading to reasonable results. A disadvantage of this choise is that for the profile of $d S / d z$ $5 \mathrm{~m}$ and for $d^{2} S / d z^{2}$ even $10 \mathrm{~m}$ are lost at the bottom and the top. While in most cases this loss does not matter at the top, sometimes it causes the lower halocline boundary $l$ (and therefore the halocline thickness $\Delta d$ ) or even the complete halocline, i.e. $d$, not to be found.

The halocline parameters were determined from the salinity profil at each station and their mean values and respective standard deviations were determined for each data set. Additionally, the halocline parameters resulting from the isobaric mean profil of salinity were determined for each data set. The results for the halocline depth $d$ and its thickness $\Delta d$ are given in Table 8 for all data sets, i.e. the mean halocline depth $\bar{d}$, the corresponding number of samples $n_{d}$ and the respective standard deviation $\sigma_{d}$, the halocline depth $d_{c}$ determined from the salinity profile at the central station, the halocline depth $d_{\bar{S}}$ determined from the isobaric mean profile of salinity, and the analogous parameters $\overline{\Delta d}, n_{\Delta d}, \sigma_{\Delta d}$, $\Delta d_{c}$, and $\Delta d_{\bar{S}}$ for the halocline thickness $\Delta d$. The analogous results $\bar{u}, n_{u}, \sigma_{u}, u_{c}, u_{\bar{S}}$ for the upper boundary $u$ of the halocline and $\bar{l}, n_{l}, \sigma_{l}, l_{c}, l_{\bar{S}}$ for its lower boundary $l$ are given in Table 9.

\subsection{First baroclinic Rossby radii}

In general, the first baroclinic Rossby radii are calculated from the profiles of the squared buoyancy or Brunt-Väisälä frequency $N^{2}$ by solving the vertical eigenvalue problem for the vertical eigenfunctions $F_{n}(z)$ and the corresponding eigenvalues $\lambda_{n}^{2}$ :

$$
\frac{d}{d z}\left(\frac{1}{N^{2}(z)} \frac{d}{d z} F_{n}(z)\right)+\lambda_{n}^{2} F_{n}(z)=0
$$


Table 8. The mean halocline depth $\bar{d}$, the corresponding number of samples $n_{d}$ and the respective standard deviation $\sigma_{d}$, the halocline depth $d_{c}$ determined from the salinity profile at the central station, the halocline depth $d_{\bar{S}}$ determined from the isobaric mean profile of salinity, and the analogous parameters $\overline{\Delta d}, n_{\Delta d}, \sigma_{\Delta d}, \Delta d_{c}$, and $\Delta d \bar{S}$ for the halocline thickness $\Delta d$ for all data sets.

\begin{tabular}{llllllllllllc}
\hline Region & Season & Data set & $\bar{d} / \mathrm{m}$ & $n_{d}$ & $\sigma_{d} / \mathrm{m}$ & $d_{c} / \mathrm{m}$ & $d_{\bar{S}} / \mathrm{m}$ & $\overline{\Delta d} / \mathrm{m}$ & $n_{\Delta d}$ & $\sigma_{\Delta d} / \mathrm{m}$ & $\Delta d_{c} / \mathrm{m}$ & $\Delta d d_{\bar{S}} / \mathrm{m}$ \\
\hline \multirow{2}{*}{$\mathrm{AB}$} & winter & MD-04 & 31 & 105 & 7 & 31 & 40 & 8 & 13 & 1 & N/A & N/A \\
& winter & MD-12 & 27 & 141 & 7 & 19 & 31 & 9 & 56 & 2 & 9 & N/A \\
BB & summer & MD-03 & 54 & 162 & 4 & 55 & 53 & 11 & 137 & 2 & 10 & 12 \\
& winter & MD-05 & 54 & 224 & 6 & 61 & 58 & 9 & 181 & 1 & 8 & 11 \\
SF & summer & MD-06 & 42 & 136 & 20 & 61 & 64 & 10 & 57 & 2 & 9 & 10 \\
& winter & MD-01 & 42 & 138 & 21 & 72 & 67 & 9 & 40 & 2 & N/A & 10 \\
& winter & MD-09 & 47 & 113 & 18 & 62 & 63 & 9 & 36 & 1 & 10 & 11 \\
EGB & summer & MD-02 & 80 & 202 & 14 & 86 & 81 & 11 & 189 & 4 & 26 & 27 \\
& summer & MD-07 & 72 & 206 & 8 & 73 & 74 & 11 & 200 & 2 & 12 & 13 \\
& summer & MD-11 & 69 & 219 & 8 & 70 & 70 & 11 & 213 & 2 & 10 & 12 \\
& winter & MD-08 & 63 & 192 & 6 & 64 & 64 & 10 & 189 & 2 & 9 & 10 \\
& winter & MD-10 & 67 & 180 & 9 & 68 & 67 & 12 & 176 & 4 & 8 & 19 \\
\hline
\end{tabular}

Table 9. The mean upper boundary $\bar{u}$ of the halocline, the corresponding number of samples $n_{u}$ and the respective standard deviation $\sigma_{u}$, the upper boundary $u_{c}$ of the halocline determined from the salinity profile at the central station, the upper boundary $u_{\bar{S}}$ of the halocline determined from the isobaric mean profile of salinity, and the analogous parameters $\bar{l}, n_{l}, \sigma_{l}, l_{c}$, and $l_{\bar{S}}$ for the lower boundary $l$ of the halocline for all data sets.

\begin{tabular}{lllllllllllll}
\hline Region & Season & Data set & $\bar{u} / \mathrm{m}$ & $n_{u}$ & $\sigma_{u} / \mathrm{m}$ & $u_{c} / \mathrm{m}$ & $u_{\bar{S}} / \mathrm{m}$ & $\bar{l} / \mathrm{m}$ & $n_{l}$ & $\sigma_{l} / \mathrm{m}$ & $l_{c} / \mathrm{m}$ & $l_{\bar{S}} / \mathrm{m}$ \\
\hline \multirow{2}{*}{ AB } & winter & MD-04 & 27 & 94 & 6 & 26 & 35 & 26 & 19 & 7 & N/A & N/A \\
& winter & MD-12 & 22 & 131 & 6 & 14 & 26 & 26 & 64 & 5 & 23 & N/A \\
BB & summer & MD-03 & 48 & 162 & 4 & 49 & 47 & 59 & 137 & 4 & 59 & 59 \\
& winter & MD-05 & 49 & 224 & 6 & 57 & 52 & 58 & 181 & 6 & 65 & 63 \\
SF & summer & MD-06 & 41 & 115 & 17 & 56 & 59 & 42 & 75 & 21 & 65 & 69 \\
& winter & MD-01 & 40 & 121 & 19 & 68 & 62 & 31 & 55 & 11 & N/A & 72 \\
& winter & MD-09 & 44 & 105 & 15 & 57 & 57 & 48 & 42 & 21 & 67 & 68 \\
EGB & summer & MD-02 & 75 & 198 & 11 & 72 & 62 & 84 & 193 & 14 & 98 & 89 \\
& summer & MD-07 & 66 & 206 & 8 & 66 & 67 & 78 & 200 & 7 & 78 & 80 \\
& summer & MD-11 & 63 & 218 & 7 & 65 & 64 & 75 & 214 & 6 & 75 & 76 \\
& winter & MD-08 & 58 & 190 & 3 & 59 & 59 & 68 & 191 & 6 & 68 & 69 \\
& winter & MD-10 & 60 & 180 & 10 & 64 & 57 & 72 & 176 & 7 & 72 & 76 \\
\hline
\end{tabular}

with the boundary conditions :

$$
\begin{aligned}
\frac{d F_{n}}{d z}(0) & =-\frac{N^{2}(0)}{g} F_{n}(0) \\
\frac{d F_{n}}{d z}(-H) & =0
\end{aligned}
$$

at the surface $(z=0)$ and at the bottom $(z=-H)$ with the upward directed vertical coordinate $z$, the depth $H$ of the water column, and the acceleration due to gravity $g$. In most cases the solutions of the vertical eigenvalue problem have to be found numerically, in particular, this applies for measured profiles of $N^{2}$ such as used for this work. For the numerical solution it is common to transform Eq. (2) to:

$\frac{d^{2}}{d z^{2}} Z_{n}(z)+\lambda_{n}^{2} N^{2}(z) Z_{n}(z)=0$

using the relation:

$\frac{1}{N^{2}(z)} \frac{d}{d z} F_{n}(z)=Z_{n}(z)$

Moreover, it is convenient to apply the rigid lid boundary condition at the surface because it suppresses the barotropic mode which is of no interest here due to the purpose to estimate the first baroclinic Rossiby radii from the profiles of $N^{2}$. In the rigid lid approximation the boundary conditions for Eq. (5) corresponding to Eqs. (3) and (4) become to:

$$
Z_{n}(0)=0
$$


$Z_{n}(-H)=0$

The first baroclinic Rossby radius $r$ is calculated from the smallest baroclinic eigenvalue $\lambda_{1}^{2}$, i.e. the inverse of the largest respective squared phase speed $c_{1}^{2}=1 / \lambda_{1}^{2}$, according to :

$r=\frac{1}{\lambda_{1}|f|}$

with the inertial frequency or Coriolis parameter $f=2 \Omega \sin (\varphi)$ calculated from the rotation rate $\Omega$ of the earth and the geographical latitude $\varphi$.

The system of Eqs. (5), (7), and (8) is solved for the measured profiles of $N^{2}$ using a finite differences approach which reduces the problem to the determination of the eigenvalues of an ordinary square matrix.

The profiles of $N^{2}$ used for the solution of the vertical eigenvalue problem were determined from the profiles of the potential density $\varrho_{\text {pot }}$ exactly in the same way as before in the context of Eq. (1) in Sect. 3.1. But the profiles of $\varrho_{\text {pot }}$ were linear interpolated before their first derivation $d \varrho_{\text {pot }} / d z$ was determined by the linear fits to avoid large data gaps within the profiles of $N^{2}$. Moreover, the resulting profiles of $N^{2}$ were constantly extrapolated to the lowest pressure level $p=1 \mathrm{dbar}$ at the surface. The depth range considered for the solution of the vertical eigenvalue problem ranges from the additional surface pressure $p=0$ to the maximum pressure $p_{\max }$ in the corresponding profiles representing $H$ in the calculations although no values of $N^{2}$ exist for these two pressures. However, the values of $N^{2}$ at the bottom and the surface are irrelevant for the calculations due to the boundary conditions according to Eqs. (7) and (8).

The first baroclinic Rossby radius $r$ was calculated from the profil of the squared buoyancy or Brunt-Väisälä frequency $N^{2}$ at each station considering the whole covered depth range of all profiles at the same station and its mean value and respective standard deviation was determined for each data set. Additionally, the first baroclinic Rossby radius resulting from the total isobaric mean profil of $N^{2}$ was calculated for each data set. The resulting first baroclinic Rossby radius $r$ is very sensitive to the depth $H$ considered for each calculation. Therefore the mean value $\bar{p}_{\max }$ of $p_{\max }$ from all profiles in each data set was calculated representing the mean depth $\bar{H}$ of the region covered by the respective field campaign and another first baroclinic Rossby radius $s$ was defined using the corresponding mean depth $\bar{H}$ as depth limit for the calculations of the first baroclinic Rossby radii in each data set. Consequently, the same calculations as for the first baroclinic Rossby radius $r$ resulting from the profiles with the corresponding total depth $H$ were done considering only the upper depth range up to $\bar{H}$ using all profiles with a maximum pressure $p_{\max } \geq \bar{p}_{\max }$. The results for the first baroclinic Rossby radii $r$ and $s$ are given in Table 10 for all data sets, i.e. the mean first baroclinic Rossby radius $\bar{r}$, the corresponding number of samples $n_{r}$ and the respective standard deviation $\sigma_{r}$, the first baroclinic Rossby radius $r_{c}$ determined from the profile of the squared buoyancy or Brunt-Väisälä frequency $N^{2}$ at the central station, and the first baroclinic Rossby radius $r \overline{N^{2}}$ determined from the isobaric mean profile of $N^{2}$ from the complete profiles acompanied by the mean depth $\bar{H}$ and the analogous parameters $\bar{s}, n_{s}, \sigma_{s}, s_{c}$, and $s \overline{N^{2}}$ for the first baroclinic Rossby radii $s$ from the limited profiles of $N^{2}$.

\section{Discussion}

The evaluation of the representativeness of the conditions at the central stations for the regional mean conditions depends on the purpose for which they are intended to be used as representative for the regional conditions. This purpose determines the accuracy needed. Basically, there are two classes of such purposes. On the one hand, there are comparative investigations between two or more regions such as a spatial monitoring of the hydrographic conditions in the whole Baltic Sea at one time. For these investigations a lower degree of accuracy in the representativeness of the conditions at the central stations is sufficient because the regional variations can be assumed to be small in relation to the interregional variations. Therefore in these investigations, the conditions at a central station are representative for the corresponding region if they agree with the respective regional mean values within the range of the typical regional variations given by the respective regional standard deviations $\sigma$. On the other hand, there are regional investigations covering just one of the regions including time series analysis for example. These investigations need a higher degree of accuracy in the representativeness of the conditions at the central stations because the deviations from the regional mean values have to be small in relation to the variations of the mean values in time. In these investigations the conditions at a central station should agree with the corresponding regional mean values within a range in the order of the uncertainty of the mean values to be representative for the region. The uncertainty of the mean values is estimated by $\sigma / \sqrt{n}$, i.e. the uncertainty of a single measurement in the estimation of the respective mean values given by the respective regional standard deviations $\sigma$ reduced by the square root of the respective number $n$ of samples as denominator.

A general evaluation of the representativeness of the conditions at the central stations for the regional mean conditions is not possible because of the different accuracies in the representativeness needed for different purposes. Following the motivation of this work, the representativeness of the conditions at the central stations is evaluated with respect to comparative monitoring purposes of the whole Baltic Sea in this article. For other purposes requiring different accuracies in the representativeness it has to be judged independently if the representativeness of the conditions at the central stations satisfies the needs of the intended investigation. However, 
Table 10. The mean first baroclinic Rossby radius $\bar{r}$, the corresponding number of samples $n_{r}$ and the respective standard deviation $\sigma_{r}$, the first baroclinic Rossby radius $r_{c}$ determined from the profile of the squared buoyancy or Brunt-Väisälä frequency $N^{2}$ at the central station, and the first baroclinic Rossby radius $r \overline{N^{2}}$ determined from the isobaric mean profile of $N^{2}$ (all from the complete profiles), the mean depth $\bar{H}$, and the analogous parameters $\bar{s}, n_{s}, \sigma_{s}, s_{c}$, and $s_{N^{2}}$ for the first baroclinic Rossby radii $s$ from the depth limited profiles of $N^{2}$ for all data sets.

\begin{tabular}{llllllllllllll}
\hline \multirow{2}{*}{ Region } & Season & Data set & $\bar{r} / \mathrm{km}$ & $n_{r}$ & $\sigma_{r} / \mathrm{km}$ & $r_{c} / \mathrm{km}$ & $r_{N^{2}} / \mathrm{km}$ & $\bar{H} / \mathrm{m}$ & $\bar{s} / \mathrm{km}$ & $n_{s}$ & $\sigma_{s} / \mathrm{km}$ & $s_{c} / \mathrm{km}$ & $s \frac{N^{2}}{2} / \mathrm{km}$ \\
\hline AB & winter & MD-04 & 3.1 & 231 & 2.0 & 5.6 & 4.7 & 40 & 2.7 & 157 & 1.4 & 4.6 & 2.9 \\
& winter & MD-12 & 5.4 & 170 & 1.6 & 6.2 & 6.5 & 41 & 5.5 & 124 & 0.6 & 5.7 & 5.4 \\
BB & summer & MD-03 & 7.2 & 176 & 1.5 & 7.9 & 9.4 & 78 & 7.1 & 110 & 0.3 & 6.9 & 7.1 \\
& winter & MD-05 & 6.9 & 240 & 1.9 & 8.1 & 9.1 & 77 & 7.2 & 129 & 0.4 & 6.7 & 7.0 \\
SF & summer & MD-06 & 2.9 & 198 & 1.7 & 6.4 & 7.1 & 56 & 2.0 & 100 & 0.2 & 2.1 & 2.0 \\
& winter & MD-01 & 2.1 & 192 & 1.9 & 6.3 & 6.8 & 58 & 1.4 & 97 & 0.6 & 1.7 & 1.4 \\
& winter & MD-09 & 2.4 & 176 & 2.2 & 6.8 & 7.2 & 57 & 1.5 & 87 & 0.8 & 0.9 & 1.6 \\
EGB & summer & MD-02 & 7.4 & 208 & 2.0 & 9.7 & 9.9 & 165 & 7.8 & 108 & 0.2 & 7.6 & 7.7 \\
& summer & MD-07 & 8.3 & 208 & 1.7 & 10.4 & 10.5 & 165 & 8.7 & 108 & 0.1 & 8.6 & 8.6 \\
& summer & MD-11 & 8.3 & 221 & 1.7 & 10.3 & 10.5 & 164 & 8.6 & 115 & 0.1 & 8.5 & 8.6 \\
& winter & MD-08 & 8.0 & 195 & 1.9 & 10.1 & 10.1 & 167 & 8.5 & 99 & 0.2 & 8.6 & 8.5 \\
& winter & MD-10 & 8.2 & 182 & 2.0 & 10.6 & 10.4 & 167 & 8.7 & 93 & 0.1 & 8.8 & 8.6 \\
\hline
\end{tabular}

the results presented in this article are helpful to support such evaluations necessary for other purposes.

\subsection{Profiles of hydrographic and physical quantities}

The Figs. 3 to 6 reveal the differences between the profiles of salinity, potential temperature, and oxygen content at the central station and the corresponding isobaric mean profiles for one summer and one winter stratification situation for each of the four regions under investigation except for the $A B$ for which no data set representing a summer stratification situation is available, see Table 2. For the winter stratification situation in the AB some discrepancies occur in all profiles in the range of the halocline with respect to the respective standard deviations. Moreover, the general shape of the profiles at the central station differs from that of the corresponding isobaric mean profiles which are more smooth due to the averaging. The same applies for all profiles corresponding to the winter stratification situation in the BB and the SF while all profiles at the central station for the summer stratification situation in the BB are in good agreement with the corresponding isobaric mean profiles in relation to the respective standard deviations. For the summer stratification situation in the SF some discrepancies in the profiles of potential temperature occur in the depth range between the seasonal thermocline and the permanent halocline. The potential temperature profile at the central station reveals some fluctuations in this depth range which are filtered out in the corresponding isobaric mean profile resulting in significant differences both in relation to the respective standard deviations and in shape. The respective profiles of salinity and oxygen content for the summer stratification situation in the SF reveal discrepancies with respect to the respective standard deviations and in shape such as found for the winter stratification situations in the AB, BB, and SF. For the EGB all profiles at the central station match the corresponding isobaric mean profile well in shape over the whole depth range with some minor differences in relation to the respective standard deviations in the depth range over the permanent halocline and below it both for the summer and the winter stratification situation.

In the Tables 6 and 7 the vertical root mean squares $r m s$ of the deviations of the profiles at the central stations from the corresponding mean profiles and the vertical mean values $\bar{\sigma}$ of the isobaric standard deviations are compared for the profiles of salinity, potential temperature, oxygen content, potential density, and squared buoyancy or Brunt-Väisälä frequency for all data sets. While the vertical root mean squares $r m s$ characterise the overall deviation of the profiles at the central station from the corresponding isobaric mean profiles, the vertical mean values $\bar{\sigma}$ of the isobaric standard deviations give a corresponding measure of the overall variations within the complete data fields. Therefore in a sense a profile at the central station can be assumed to represent the corresponding isobaric mean profile well, if the vertical root mean squares rms for this profile is small compared to the corresponding vertical mean value $\bar{\sigma}$ of the respective isobaric standard deviations.

The results given in Table 6 reveal that this condition is meet by most data fields listed there. The data fields from the EGB meet this condition most clearly, in particular the data fields of salinity and, consequently, the data fields of potential density which is mainly determined by salinity in the Baltic Sea. Accordingly, the fewest exceptions are found for the data fields from the EGB, the most for the data fields from the AB. All exceptions belong to data fields representing a winter stratification situation. In spite of the standardised 
estimates of the isobaric overall deviations and variations the good matches for the data fields from the EGB partly may be attributed to the smaller fraction of the total depth of the profiles covered by the halocline in which the major isobaric variations and, consequently, deviations occur, see Figs. 3 to 6. However, even if the good matches for the data sets from the EGB solely resulted from a smaller fraction of the total depth covered by the halocline, this result would be reasonable because the profiles at the central station in the EGB would be more representative for a larger depth fraction of the profiles and, consequently, would have a better overall match than the profiles from the central stations in the other regions.

For all data fields of the squared buoyancy or BruntVäisälä frequency presented in Table 7 the vertical root mean squares $r m s$ for the profil at the central station is smaller than the corresponding vertical mean value $\bar{\sigma}$ of the isobaric standard deviations by about three orders of magnitude. Therefore the condition of small vertical root mean squares $r m s$ for the profil at the central station compared to the corresponding vertical mean value $\bar{\sigma}$ of the isobaric standard deviations is meet by the data fields of the squared buoyancy or BruntVäisälä frequency $N^{2}$ most clearly. The smoothing of the profiles of $N^{2}$ within their calculation due to the estimation of the derivation $d \varrho_{\text {pot }} / d z$ can not be assumed to cause these good matches. The according minimal noise reduction necessary to get realistic profiles of $N^{2}$ can not be expected to change the order of magnitude of the isobaric standard deviations or the deviations between the profiles at the central stations and the corresponding isobaric mean profiles of $N^{2}$. The large vertical mean values $\bar{\sigma}$ of the isobaric standard deviations may be attributed to large scale inclinations of the seasonal thermocline and the permanent halocline resulting in large isobaric variations of the squared buoyancy or BruntVäisälä frequency.

\subsection{Parameters of the permanent halocline}

The depth $d$ of the permanent halocline, its upper and lower boundaries $u$ and $l$, respectively, and its thickness $\Delta d=l-u$ were determined from the salinity profile at each station and from the corresponding isobaric mean profile for each data set. The results in Tables 8 and 9 reveal a satisfactory agreement of the result for each halocline parameter determined from the salinity profile at the central station, the respective result determined from the isobaric mean profile of salinity, and the respective mean value over all stations with respect to the respective standard deviation for most data sets. The most significant differences are found in a data field from the SF representing a winter stratification situation for the depth of the halocline and its boundaries and in a data field from the EGB representing a summer stratification situation for the halocline thickness. In spite of this, the overall agreement of the various halocline parameters is the best for the EGB as can be assumed from the discussion in Sect. 4.1.
The worst overall agreement of the various halocline parameters is found in the SF according to the numerous significant differences in the corresponding data fields, although the standard deviations of all halocline parameters other than the halocline thickness $\Delta d$ are twice and more times larger for the data sets from the SF than for the data sets from the other regions. In general, the results for the boundaries of the halocline and, consequently, for its thickness determined from the salinity profile at the central station match the respective mean values somewhat better with respect to the respective standard deviations than the respective results determined from the isobaric mean profiles of salinity due to the smearing of a large scale inclined halocline caused by the isobaric spatial averaging.

\subsection{First baroclinic Rossby radii}

The first baroclinic Rossby radii $r$ and $s$ were calculated from the profiles of the squared buoyancy or Brunt-Väisälä frequency at each station and from the corresponding isobaric mean profile for each data set. While the whole depth range covered by the profiles was considered for the calculations of the first baroclinic Rossby radii $r$, only the depth range from the surface to the mean depth $\bar{H}$ of all profiles in each data set was taken into account for the corresponding calculations of the first baroclinic Rossby radii $s$. Therefore the first baroclinic Rossby radii $s$ could have been calculated only for profiles reaching at least as deep as the mean depth $\bar{H}$ for the corresponding data set. The results in Table 10 reveal a satisfactory agreement between the first baroclinic Rossby radii $r$ calculated from the profile of squared buoyancy or Brunt-Väisälä frequency at the central station and those calculated from the corresponding isobaric mean profile for all data sets. However, they reveal significant differences between each of both and the corresponding mean value of the first baroclinic Rossby radii $r$ over each data set in relation to the respective standard deviations for most data sets. The discrepancies are most striking for the data sets from the SF. Obviously, the reason for these differences is the dependence of the resulting first baroclinic Rossby radii on the considered depth $H$ which varies considerably within each data set for the first baroclinic Rossby radii $r$, since the whole depth range covered by each profile is taken into account in the corresponding calculation. The dependence of the resulting first baroclinic Rossby radii $r_{b c}$ on the considered depth $H^{\prime}$ is illustrated in Fig. 7 for the profile of the squared buoyancy or Brunt-Väisälä frequency at the central station and the corresponding isobaric mean profile of the data set MD-07 representing a summer stratification situation in the EGB. Additionally, the resulting first baroclinic Rossby radii $r$ are plotted which were calculated from the corresponding complete profile reaching to the depth $H$ at each station. The Fig. 7 suggests that the results given in Table 10 concerning the first baroclinic Rossby radii $r$ calculated from the complete profiles are reasonable in spite of the 
significant differences found between the mean values of the first baroclinic Rossby radii $r$ and those calculated from the corresponding profile of squared buoyancy or Brunt-Väisälä frequency at the central station or the corresponding isobaric mean profile.

In contrast to the first baroclinic Rossby radii $r$, the first baroclinic Rossby radii $s$ are calculated from the profiles of the squared buoyancy or Brunt-Väisälä frequency taking into account the same depth range for all profiles which is chosen to the depth range from the surface to the mean depth $\bar{H}$ of all profiles in each data set. Therefore no differences of the calculated first baroclinic Rossby radii $s$ can result from different depth ranges considered for single profiles. Consequently, the results given in Table 10 for the first baroclinic Rossby radii $s$ reveal a high degree of agreement between all the first baroclinic Rossby radii $s$ calculated from the profile of the squared buoyancy or Brunt-Väisälä frequency at the central station, those calculated from the corresponding isobaric mean profile, and the corresponding mean value of the first baroclinic Rossby radii $s$ over each data set in relation to the respective standard deviations for all data sets. The only two exceptions occur for the first baroclinic Rossby radii $s$ calculated from the profile of squared buoyancy or Brunt-Väisälä frequency at the central station of the two data sets representing a winter stratification situation, one from the $\mathrm{AB}$ and one from the $\mathrm{BB}$. Remarkably, all of the three first baroclinic Rossby radii $s$ given in Table 10 for each data set also match the mean value of the corresponding first baroclinic Rossby radii $r$ calculated from the complete profiles of squared buoyancy or Brunt-Väisälä frequency quiet well for all data sets.

\section{Conclusions}

Although some discrepancies between the profiles at the central stations and the corresponding isobaric mean profiles exist, the profiles of salinity, potential temperature, oxygen content, potential density, and squared buoyancy or BruntVäisälä frequency at the central station match the corresponding isobaric mean profiles in a satisfactory way with respect to the respective standard deviations. The best matches are found for the squared buoyancy or Brunt-Väisälä frequency which may be attributed to large scale inclinations of the seasonal thermocline and the permanent halocline resulting in large isobaric variations of the squared buoyancy or Brunt-Väisälä frequency. With respect to the regions the best agreements are found in the EGB, the worst in the AB. Furthermore, the best agreements in the EGB are found for salinity irrespectively of the stratification situation and, consequently, for potential density which is mainly determined by salinity in the Baltic Sea. The worst matches in the AB are also revealed for salinity and potential density.

The depth, thickness, upper, and lower boundaries of the permanent halocline are represented well by the salinity pro-

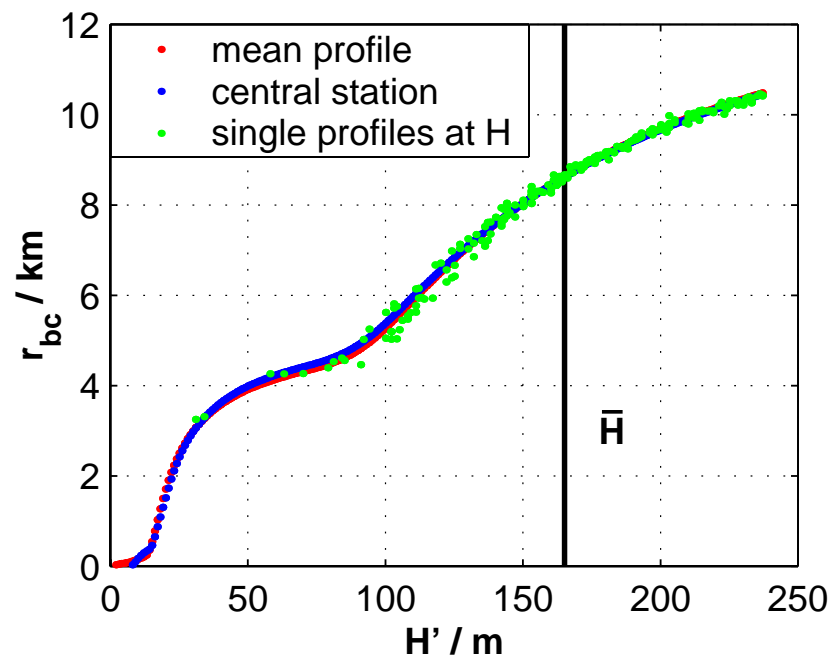

Fig. 7. Dependence of the resulting first baroclinic Rossby radii $r_{b c}$ on the considered depth $H^{\prime}$ for the profile of the squared buoyancy or Brunt-Väisälä frequency at the central station and the corresponding isobaric mean profile of the data set MD-07 representing a summer stratification situation in the EGB and the resulting first baroclinic Rossby radii $r$ calculated from the corresonding complete profile reaching to the depth $H$ at each station.

files at the central stations compared to both the respective parameters determined from the isobaric mean profiles of salinity and the respective mean values of the parameters determined at each station in the data field. This result is according to the results for the salinity profiles and applies for all of the four regions and both summer and winter stratification situations.

The first baroclinic Rossby radii calculated from the profil of squared buoyancy or Brunt-Väisälä frequency at the central station are in good agreement with the first baroclinic Rossby radii calculated from the corresponding isobaric mean profile and the mean value of the first baroclinic Rossby radii calculated from the corresponding profiles at each station in the region, if the depth range taken into account for the calculations ranges from the surface to the mean depth of the region. This applies for all of the four regions and both summer and winter stratification situations. Moreover, the first baroclinic Rossby radii calculated from the depth limited profil of squared buoyancy or Brunt-Väisälä frequency at the central station match the mean values of the first baroclinic Rossby radii calculated from the corresponding complete profiles at all stations in the region fairly well in all cases investigated.

Overall, the regional characteristics of the investigated quantities and parameters are represented well by the hydrographic measurements at the central stations in the four regions of the Baltic Sea considered in this work. In particular, the observations at the central stations of the $\mathrm{AB}, \mathrm{BB}, \mathrm{SF}$, and EGB seem to be usefull for comparisons between these 
regions in spite of the occuring differences between the spatial mean states and the observations at the central stations. However, it is emphasised again that the representativeness of the conditions at the central stations for the regional mean conditions is evaluated with respect to comparative monitoring purposes of the whole Baltic Sea in this article. Other purposes such as regional studies covering just one of the regions require different accuracies in the representativeness and, consequently, this evaluation may be invalid for other purposes. Therefore the required accuracy in the representativeness has to be determined for each purpose separately and the results presented in this article may be used to evaluate the representativeness of the conditions at the central stations according to the needs of the intended investigation.

Acknowledgements. The data used for this work was acquired in the framework of the German-Russian project MESODYN. The German contribution of MESODYN was supported by the Federal Ministry of Education, Scientific Research and Technology of Germany.

Edited by: K. Döös

\section{References}

Bergström, S. and Carlsson, B.: River Runoff to the Baltic Sea: 1950-1990, AMBIO, 23, 280-287, 1994.

Feistel, R.: A new extended Gibbs thermodynamic potential of seawater, Progress in Oceanography, 58, 43-115, 2003, Corrigendum, 61, 99, 2004.

Feistel, R.: Numerical implementation and oceanographic application of the Gibbs thermodynamic potential of seawater, Ocean Sci., 1, 9-16, 2005, http://www.ocean-sci.net/1/9/2005/.
Fennel, W., Seifert, T., and Kayser, B.: Rossby radii and phase speeds in the Baltic Sea, Continental Shelf Res., 11, 23-36, 1991.

Hagen, E. and Feistel, R.: Observations of low-frequency current fluctuations in deep water of the Eastern Gotland Basin/Baltic Sea, J. Geophys. Res., 109, C03044, doi:10.1029/2003JC002017, 2004.

HELCOM: Water balance of the Baltic Sea, Baltic Sea Environment Proceedings No. 16, Baltic Marine Environment Protection Commission, Helsinki, Finland, 1986.

Hennemuth, B., Rutgersson, A., Bumke, K., Clemens, M., Omstedt, A., Jacob, D., and Smedman, A.-S.: Net precipitation over the Baltic Sea for one year using models and data-based methods, Tellus, 55A, 352-367, 2003.

Kõuts, T. and Omstedt, A.: Deep water exchange in the Baltic Proper, Tellus, 45A, 311-324, 1993.

Lindau, R.: Energy and water balance of the Baltic Sea derived from merchant ship observations, Boreal Environ. Res., 7, 417424, 2002.

Matthäus, W.: Charakteristische Eigenschaften von Stagnationsperioden im Tiefenwasser der Ostsee, Beiträge zur Meereskunde, 55, 39-53, 1986.

Omstedt, A. and Axell, L. B.: Modeling the seasonal, interannual, and long-term variations of salinity and temperature in the Baltic proper, Tellus, 50A, 637-652, 1998.

Omstedt, A. and Nohr, C.: Calculating the water and heat balances of the Baltic Sea using ocean modelling and available meteorological, hydrological and ocean data, Tellus, 56A, 400-414, 2004.

Omstedt, A., Elken, J., Lehmann, A., and Piechura, J.: Knowledge of the Baltic Sea physics gained during the BALTEX and related programmes, Progress in Oceanography, 63, 1-28, 2004.

Rodhe, J.: The Baltic and North Seas: A Process-orientated Review of the Physical Oceanography, in: The Sea, edited by: A. R. Robinson and K. H. Brink, 11, 24, 699-732, John Wiley \& Sons, Inc., New York, 1998. 